\title{
Origins to Outcomes: A Role for Extracellular Vesicles in Precision Medicine
}

\author{
John Savage ${ }^{\mathrm{ab},}$, Ciaran Manus Maguire ${ }^{\mathrm{b}, \mathrm{c}}$, Adriele Prina-Mello, ${ }^{\mathrm{b}, \mathrm{c} *}$ \\ ${ }^{a}$ School of Medicine, Trinity College Dublin, Dublin 2, Ireland. \\ ${ }^{b}$ Laboratory for Biological Characterization of Advanced Materials (LBCAM), Department of Clinical \\ Medicine, Trinity Translational Medicine Institute, School of Medicine, Trinity College Dublin, Dublin, Dublin \\ 8 , Ireland. \\ ${ }^{c} A M B E R$ Centre, CRANN Institute, Trinity College Dublin, Dublin 2, Ireland.
}

Submitted: March 8, 2018;

Accepted: April 20, 2018

Published: May 11, 2018

\begin{abstract}
Extracellular vesicles (EVs) are of great interest in biological research, and though they are a relatively recent discovery, they have rapidly shown great potential for use in clinical applications. The various techniques used in EV isolation along with their respective strengths, weaknesses, and potential for downstream applications are outlined here. A brief description of the different approaches in exosome characterisation are subsequently described. It has been highlighted that despite the recent developments in these processes, there is still a great deal of refinement to be made. EVs are produced by almost all cell types, found in many biological samples, and are implicated in multiple biological processes including cargo trafficking, cell-cell communication, and signal transduction. The presence of these EVs and their varied cargo in a biological sample can be indicative in disease diagnosis, and guide precision medicinebased approaches to disease management. EVs have been reported to act in the benefit of the cell through moderating repair and regeneration, but they can also act to the detriment of the cell through increased tumorigenesis and metastasis. This duality is intriguing as it can allow for the use of EVs in distinct therapeutic approaches and displays their versatility in potential downstream applications. In this review, examples of the cellular roles of EVs and their applications in pathological and regenerative contexts are explored. In reviewing some of the developments made in recent times, EVs are shown to be very promising both in diagnostic and therapeutic approaches. ${ }^{i}$
\end{abstract}

Keywords:

Extracellular vesicles, regenerative medicine, biomarkers, EV isolation, EV characterisation, cancer

\section{Rationale and Purpose}

Increasing body of evidence suggests that healthcare, medical decisions, practices, and treatments should be tailored towards individual patients to improve their outcomes, and there is now need for precise and personalised medicine. Extracellular vesicles are produced by almost all cell types, found in many biological samples and are implicated in multiple biological processes including cargo trafficking, cell-cell communication and signal transduction. The presence of these vesicles and their varied cargo in a biological sample can be indicative in disease diagnosis, and guide precision medicine-based approaches to disease management. EVs have been reported to act in the benefit of the cell

\footnotetext{
* Ussher Assistant Professor / LBCAM Director, Trinity Translational Medicine Institute (TTMI), Department of Clinical Medicine, School of Medicine, Trinity College Dublin, the University of Dublin; Contact details: Tel.+35318963259/3260; E-mail:prinamea@tcd.ie
} 
through moderating repair and regeneration, but they can also act to the detriment of the cell through increased tumorigenesis and metastasis. This duality is intriguing as it can allow for the use of EVs in distinct therapeutic approaches and displays their versatility in potential downstream applications. In this review, examples of the cellular roles of EVs and their applications in pathological and regenerative contexts are explored.

\section{Summary of Relevant Literature}

Extracellular vesicles (EVs) can have varied cellular roles and they have been highlighted for use as potential clinical biomarkers [1]. These small nano- to sub-micron sized lipid membrane enclosed structures, comprise varying contents, and are produced by almost all cell types [2]. EVs were initially observed as a product of reticulocytes that were released into the extracellular environment. The study was regarding transferrin receptor endocytosis and the unintended outcome of revealing the production and secretion of EVs that contained transferrin receptors [3].

EVs were found to form after several intracellular processing steps. Initially, intraluminal vesicles form and are contained in multivesicular endosome (MVE) bodies. These MVE bodies subsequently are trafficked to and fuse with the plasma membrane of the cell. These MVE bodies range between 0.5 and $1 \mu \mathrm{m}$ in diameter. Their fusion with the plasma membrane resulted in the release of multiple EVs of a diameter between 40 and $100 \mathrm{~nm}$ [4]. It has also been reported that certain EV subsets can occur at lipid raft regions which are associated with high levels of cholesterol and sphingomyelin [5].

Upon closer examination, it was found that these EVs displayed composition and function characteristic of their cell of origin. These EVs were thought to be utilised as a mechanism of aiding cell maturation, along with the removal of excess cellular materials [4]. However, it has been demonstrated in subsequent research that they can possess diverse contents. The contents of the different EV subtypes can be catalogued in various online databases, including ExoCarta [6], EVpedia [7] and Vesiclepedia [8].

There are far more roles for these EVs than was previously thought, and this has been discerned considering the varied contents that they can possess. They have been shown to have roles in many aspects of specific pathological processes, which are deleterious to the host. These can include immune suppression, [ cellular communication through protein trafficking to drive excessive proliferation, [ and they can increase cancer aggressiveness through micro RNA (miRNA) trafficking. [11]

However, it has also been documented that EVs, particularly those released by stem cells, can have regenerative roles in a variety of disease states and tissues. Examples of this can be found in liver tissue regeneration via cargo shuttling between cells, [ peripheral nervous system repair, through modifying cellular growth,[ skeletal muscle regeneration through induction of stem cell differentiation [14], cardio protection, [ and cardiac regeneration post injury, through alterations to target cell signalling. [16]

An important caveat that should be considered in the study of EVs is that they can be produced by cells and they can be divided into many categories, including exosomes, apoptotic bodies and microvesicles [2]. It has also been shown that even among the EVs produced from a single cell type of origin, that distinct subpopulations can occur. These subpopulations can demonstrate different marker proteins, internal cargo, and distinct downstream effects on gene expression. This reveals an additional layer of complexity in EV study, but may allow for more avenues of investigation to be pursued regarding downstream applications, including therapeutics [17]. Similarly, with the study of the heterogeneous subpopulations of EVs produced, insight may be granted into how EVs can impact so many diverse functions and how they can aid in tumorigenesis [18].

With this in mind, EVs can be utilised as biomarkers for cancers of various tissues and various diagnostic methods have been designed to utilise EVs in a clinical context, enabling personalised and precision medicine based approaches [19]. There are currently pilot studies for $\mathrm{EV}$ use as diagnostic markers [20,21], along with early phase 1 clinical trials regarding EV use for regenerative applications [22] and as potential post-intervention prognostic markers in disease states [23] being conducted. EVs are also being 
used as vehicles in different therapies including small interfering RNAs (siRNAs) [24] and drug delivery [25]. This has been visually summarised in Figure 1.

Considering their manifold features and broad applicable potential, the study of EVs is a complex field that involves the discussion of many aspects such as those below.

\section{EV Isolation}

Considering the range of EVs that can be produced by a single cell type and the size of EVs produced, much consideration should be taken regarding the isolation method utilised. There are many methods that can be implemented, yet the impact of the isolation procedures on the EVs should be taken into account. It has been suggested that the EVs may be affected by the acquisition steps taken, though it can be difficult to determine the exact effects and how best to alleviate them [28]. EVs can be isolated broadly speaking, based on size and/or affinity-based methods. A summary of these methods and their characteristics have been outlined in Table I, II, and III.

A typical method of EV isolation that can be implemented considering their size is differential ultracentrifugation. This is considered the gold standard of EV isolation methods, yet there are certain contentions regarding the validity of that statement [29]. The method of differential centrifugation can involve repeated rounds of centrifugation that use increasing centrifugal force for each round, gradually removing larger unwanted materials from a sample. It has been shown that with considerations for the type of rotor, centrifuge radius, and centrifugation duration, a more optimal protocol can be developed depending on the size of the structure of interest [30].
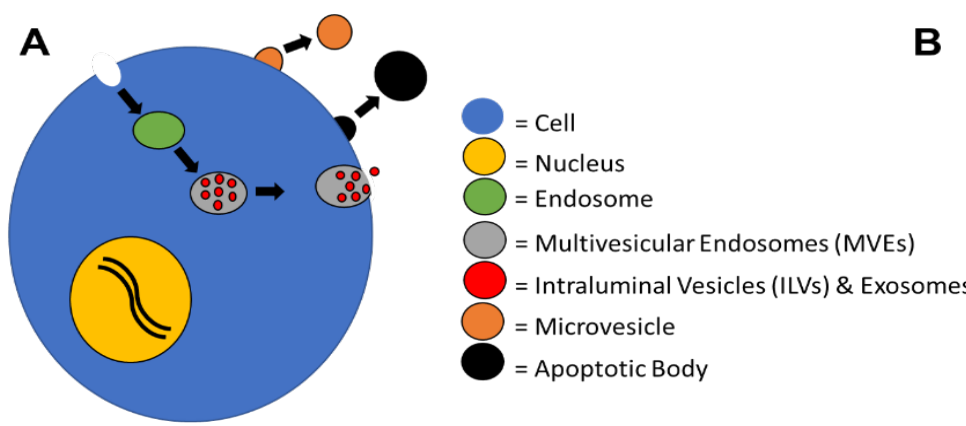

C

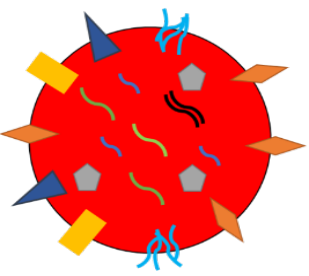

Green $=$ mRNA.

Blue $=$ miRNA.

Black $=$ DNA.

Grey $=$ Protein

Orange $=$ Carbohydrate

Yellow $=$ Tetraspanin e.g. CD9/CD81

Navy $=$ Receptor

Turquoise $=$ Lipid e.g. Ceramide
B

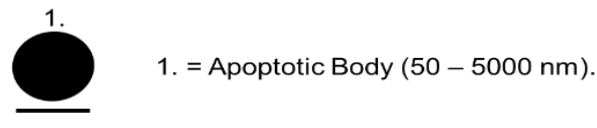

2.

2. $=$ Microvesicle $(50-2000 \mathrm{~nm})$.

3.

ㅇ. $\quad$ Exosome $(30-100 \mathrm{~nm})$.

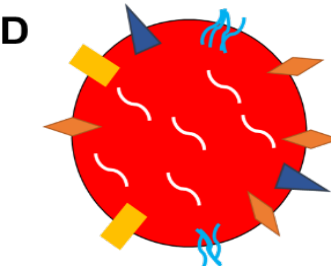

White $=$ siRNA

Orange $=$ Carbohydrate

Yellow $=$ Tetraspanin

Navy $=$ Receptor

Turquoise $=$ Lipid

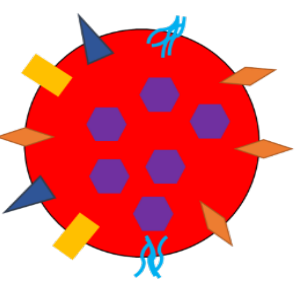

Purple $=$ Drug

Orange $=$ Carbohydrate

Yellow $=$ Tetraspanin .

Navy $=$ Receptor

Turquoise = Lipid.

Figure 1. A visual representation of EV biogenesis, the differing sorts of EVS, EV contents and how they can be engineered. (A) $A$ visual summary of the cellular processes that compose EV biogenesis, figure adapted from [26]. (B) A comparison of EV sizes including apoptotic bodies, microvesicles, and exosomes, sizes taken from [27]. (C) Exosome structure and composition in greater detail, figure adapted from [26]. (D) Potential EV applications including cargo modification with siRNAs and drugs. 
An alternative method that can be conducted is that of ultrafiltration. Ultrafiltration has been reported to produce a higher concentration of EVs $<100 \mathrm{~nm}$ than the differential ultracentrifugation method and does so in a fraction of the time required [29]. Ultrafiltration can use high pressure gas with a filter, or a centrifugation step with a filter, rather than differential centrifugation. It has been contended that the repeated centrifugation steps that are required for the ultracentrifugation method can potentially result in damage to the EVs. The ultrafiltration circumvents this issue quite neatly using the filter [29]. However, filter clogging can occur and the pressure-based approach can damage the EVs isolated [31].

Size Exclusion Chromatography (SEC) is a method that has been developed as an alternative to the ultracentrifugation method. It can allow for the isolation of singlet EVs and inhibits vesicle aggregation. This method has been reported to minimise contamination and preserve the biological function of the EVs more than ultracentrifugation methods [32]. The EV acquisition rate can be relatively slow [29] and columns typically require optimisation, depending on the application [32].

A recent discovery in the field of EV isolation, is the utilisation of acoustics to isolate EVs from a cell culture medium sample containing a heterogeneous population of EVs. It has shown a high degree of specificity, being able to separate vesicles differing in sizes by $100 \mathrm{~nm}$. An additional feature is that the filter can be adjusted electronically [33]. This technology still requires further development and investigation, yet it is a technique that has shown promise. In conjunction with chip-based technologies, it can be used on complete biological samples with potential for automation and cost-effectiveness in the future [34].

Polyethylene Glycol (PEG) is a substance that has been used in the isolation of viral particles, yet it has been shown to have potential in EV isolation [35]. One aspect that should be considered is that PEG can coat EVs with films, which can inhibit efforts to observe individual EVs using Particle Tracking Analysis (PTA) and the PEGylated EVs can tend to form aggregates [36].

Preparations of commercially available precipitation kits such as ExoQuick ${ }^{\mathrm{TM}}$ (System Biosciences, USA) and Exo-spin ${ }^{\mathrm{TM}}$ (Cell Guidance Systems, UK) can also be utilised to isolate EVs from a sample. However, there can be issues regarding contamination and a lack of purity. It has been suggested that a purification procedure post precipitation may minimise this impurity issue. This impurity issue is particularly evident on comparison with the SEC based Izon qEV column with OptiPrep ${ }^{\mathrm{TM}}$ (iZon Science, New Zealand) approach [29].

Microfluidic chips can be used as a means of $\mathrm{EV}$ isolation through both size-based and affinity-based means [37]. It has been observed that affinity-based microfluidic chips allow for the acquisition of EV populations with a higher degree of purity than the size-based methods [38]. As they are in their early development stages, there is a lack of standardisation. Additionally, their low sample yield can make downstream analyses problematic [31]. Microfluidic chips are currently limited to low-throughput approaches and require specialised equipment [36].

Magnetic beads can be used to isolate EVs. They remove the necessity of pre-enrichment steps and can allow for high-purity EVs to be obtained from a sample, while reducing the amount of handling and manipulation of the EVs to be isolated. It has been shown that the beads are typically used with only one marker-specific antibody associated (e.g., CD9 or CD81) to a set of beads [39]. In order for this method to be successfully applicable, EV surface markers must be established as present in the sample [31]. The capability of the magnetic beads to isolate EVs can be limited in this way as EVs can possess many differing surface markers. The levels of these marker proteins can vary depending on source cell type and disease state [40]. Panels of $\mathrm{EV}$-associated proteins can be tested for to isolate and characterise EVs [41]. 
Table I: Microfluidic chips can be utilised as either as a size-based or an affinity-based approach for EV isolation, image adapted from [18].

\begin{tabular}{c|l}
\hline Method of Isolation & \\
\hline Advantages & $\begin{array}{l}\text { Can be used for size-based and affinity-based EV isolation methods } \\
\text { [38]. } \\
\text { Affinity-based approaches can isolate EVs of high purity and can be } \\
\text { used with very small sample volumes [38]. }\end{array}$ \\
\hline The size-based chip approach can result in a low degree of purity [38]. \\
Lack of standardisation between methods [31]. \\
Requires specialised equipment and restricted to low throughput [36]. \\
\hline Cost
\end{tabular}

An alternative method of EV isolation and characterisation is through use of lectin coated bead induced aggregation of EVs, based on the presence of EV surface carbohydrates [42]. It has been developed to address the issue that EVs can have variable expression of their surface marker proteins. Different cell types and cancer cell lines have characteristic surface carbohydrate expression patterns and through use of lectins appropriate to the carbohydrates in question, EVs may be isolated. EVs, upon isolation from biological samples (e.g., urine) can undergo subsequent downstream analysis and be used in certain diagnostic capacities [43], yet low levels of EV isolation have been observed [42]. 
Table II. Respective advantages, disadvantages, costs, applications, and schematics of various EV isolation methods. Size-based approaches for EV isolation. The image for ultracentrifuga-tion was adapted from [31], the image for SEC is adapted from [32], the image for acoustic filtering is adapted from [34].

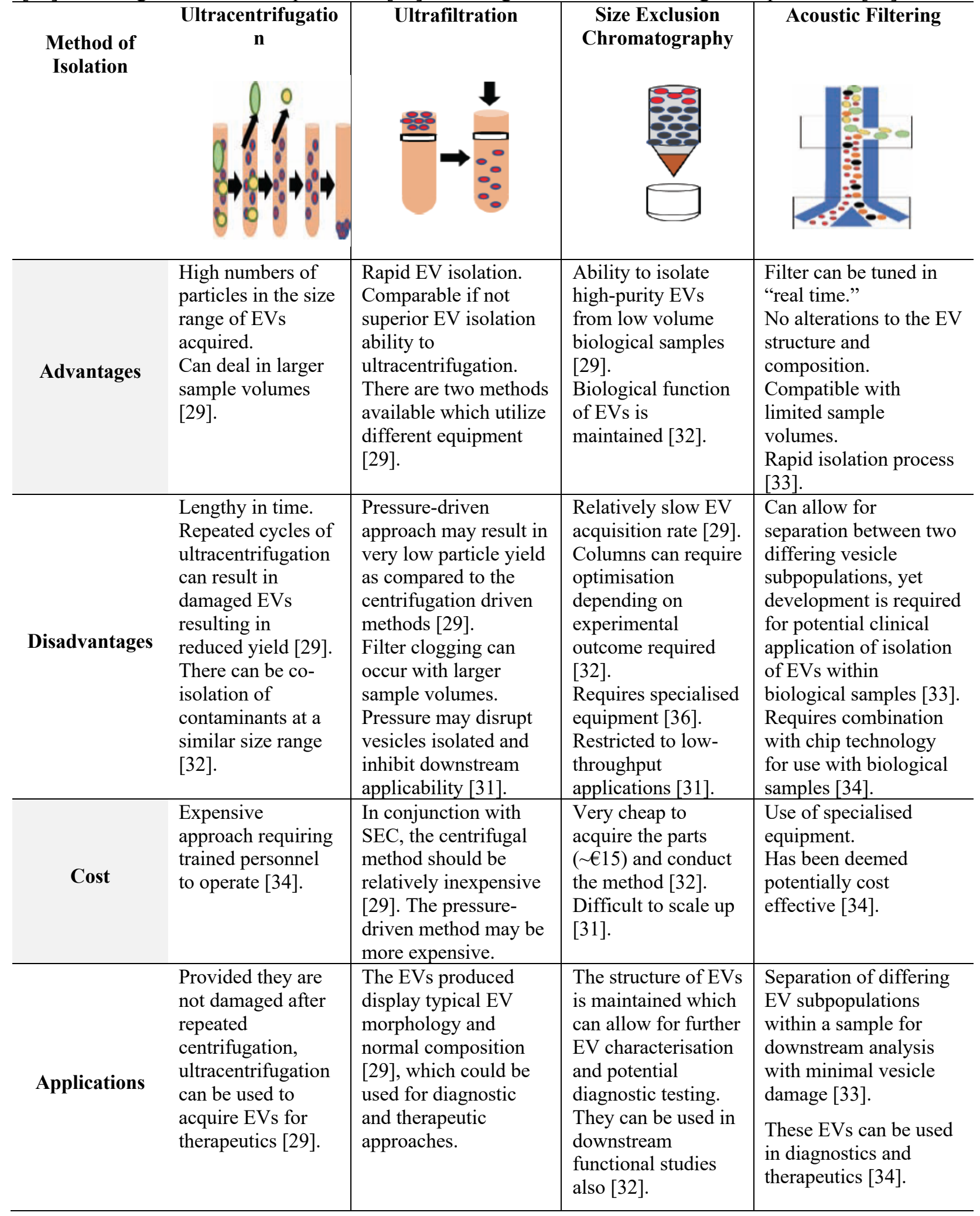


Table III: Affinity-based approaches for EV isolation. Image for magnetic beads adapted from [38].

\begin{tabular}{|c|c|c|c|c|}
\hline \multirow[b]{2}{*}{$\begin{array}{l}\text { Method of } \\
\text { isolation }\end{array}$} & \multirow[t]{2}{*}{ Magnetic Beads } & \multirow[t]{2}{*}{ Precipitation Kits } & $\begin{array}{l}\text { Polyethylene } \\
\text { Glycol (PEG) }\end{array}$ & \multirow[t]{2}{*}{$\begin{array}{c}\text { Lectin Based } \\
\text { Agglutination }\end{array}$} \\
\hline & & & 8 & \\
\hline Advantages & $\begin{array}{l}\text { Can be used to target } \\
\text { EVs using specific } \\
\text { antibodies for } \\
\text { specific antigens. } \\
\text { Potential for } \\
\text { automation [39]. }\end{array}$ & $\begin{array}{l}\text { Can be used with large } \\
\text { samples [38]. } \\
\text { Optimised protocol } \\
\text { provided by the } \\
\text { manufacturer. } \\
\text { Contaminants can be } \\
\text { removed by } \\
\text { precipitation post } \\
\text { purification [29]. }\end{array}$ & $\begin{array}{l}\text { Can be used with } \\
\text { large sample } \\
\text { volumes. } \\
\text { Can be used with } \\
\text { complete biological } \\
\text { samples. } \\
\text { EV components are } \\
\text { preserved [35]. }\end{array}$ & $\begin{array}{l}\text { EVs express surface } \\
\text { carbohydrates which } \\
\text { can be bound by } \\
\text { corresponding } \\
\text { lectins. } \\
\text { This method can be } \\
\text { applied to complete } \\
\text { biological samples } \\
\text { [43]. }\end{array}$ \\
\hline Disadvantages & $\begin{array}{l}\text { Variability in } \\
\text { expression of EV } \\
\text { surface markers. } \\
\text { Multiple magnetic } \\
\text { beads specific for } \\
\text { each marker are } \\
\text { needed if multiple } \\
\text { antigens are under } \\
\text { investigation [44]. }\end{array}$ & $\begin{array}{l}\text { Requires overnight } \\
\text { precipitation. } \\
\text { Potential for co- } \\
\text { isolation of larger } \\
\text { contaminants. } \\
\text { Commercially available } \\
\text { kits reported to have } \\
\text { differing degrees of } \\
\text { efficacy. } \\
\text { Requires a post } \\
\text { purification } \\
\text { precipitation step [29]. }\end{array}$ & $\begin{array}{l}\text { Requires an } \\
\text { ultracentrifugation } \\
\text { step [35]. } \\
\text { EVs produced } \\
\text { typically aggregate } \\
\text { which could restrict } \\
\text { cell endocytosis } \\
\text { [36]. }\end{array}$ & $\begin{array}{l}\text { Lacks } \\
\text { standardisation. } \\
\text { Discovery of optimal } \\
\text { lectins and their } \\
\text { respective } \\
\text { combinations } \\
\text { required [43]. } \\
\text { Reduced EV } \\
\text { isolation capability } \\
\text { compared to } \\
\text { antibodies } \\
\text { conjugated to } \\
\text { magnetic beads [42]. }\end{array}$ \\
\hline Cost & $\begin{array}{l}\text { Magnetic beads are } \\
\text { inexpensive, cost } \\
\text { related to the } \\
\text { antibodies used. }\end{array}$ & $\begin{array}{l}\text { Relatively expensive } \\
\text { approach based on the } \\
\text { number of samples [36]. }\end{array}$ & $\begin{array}{l}\text { Similar setup costs } \\
\text { to the } \\
\text { ultracentrifugation } \\
\text { method. } \\
\text { Cheaper alternative } \\
\text { to the commercially } \\
\text { available } \\
\text { precipitation kits } \\
\text { [35]. }\end{array}$ & $\begin{array}{l}\text { Relatively cost- } \\
\text { effective approach } \\
\text { [43]. }\end{array}$ \\
\hline Applications & $\begin{array}{l}\text { Structure of EVs is } \\
\text { maintained and can } \\
\text { be used in } \\
\text { characterisation and } \\
\text { downstream analysis } \\
\text { of EVs [44]. }\end{array}$ & $\begin{array}{l}\text { They can be utilized for } \\
\text { EV content } \\
\text { characterisation and are } \\
\text { beneficial in diagnostic } \\
\text { applications [31]. }\end{array}$ & $\begin{array}{l}\text { The EVs isolated } \\
\text { can be used for } \\
\text { diagnostic } \\
\text { applications[35]. } \\
\text { May not be usable } \\
\text { for therapeutics due } \\
\text { to aggregation that } \\
\text { can occur [36]. }\end{array}$ & $\begin{array}{l}\text { EVs isolated can } \\
\text { undergo } \\
\text { characterisation. } \\
\text { Their cargo can be } \\
\text { analysed for } \\
\text { diagnostic potential. } \\
\text { There may be use of } \\
\text { them for therapeutic } \\
\text { applications [43]. }\end{array}$ \\
\hline
\end{tabular}

\section{EV Characterization}

EVs can be characterised and subtyped based on a number of key parameters including their size (typically $<100 \mathrm{~nm}[45]$ ) and their surface markers. Due to their small size, conventional light microscopy is insufficient to identify EVs, 
and as such more advanced and higher resolution techniques are required. Transmission electron microscopy can be used for the characterisation of EVs based on morphological features. It has been suggested however, that the sample preparation of EVs for the electron microscopy examination can in fact, induce the distinctive cup-like morphology that has been associated with EVs [31]. There are other electron microscopy methods such as cryo-electron microscopy which have been reported to maintain EV spherical structure [46]. A form of microscopy which has been applied to EV characterisation is Atomic Force Microscopy (AFM). This can allow for the examination of height and diameter of multiple EVs under observation and preserves their spherical structure. AFM can be conducted without fixation, staining, or labelling, which is thought to aid in the preservation of the natural structure of the EV [5].

There are additional EV characterisation methods that focus more on the presence of surface markers and EV composition. The Bradford protein assay can be conducted to assess the level of proteins present in a sample. This can be used to determine the concentration of $\mathrm{EV}$ proteins in a sample acquired using a number of methods [47]. However, the Bradford assay is not selective, and the presence of contaminating proteins could generate misrepresentative results unless purification is conducted prior to the Bradford protein assay [48].

The presence of characteristic EV marker proteins can be demonstrated through use of Western Blotting using antibodies specific to EV marker proteins of interest [40]. A number of EV surface markers can be tested for, such as Heat Shock Protein-90 (HSP-90), CD86, and LAMP-1 [47]. However, some studies have contended that certain markers can be inconsistently present, including TSG-101 and CD63. The high levels of the exosome marker proteins CD9 and CD81 observable across cancer cell lines and differing tissues could suggest for their use as loading controls in EV Western Blotting [40].

A method that is becoming increasingly more prevalent in characterising EVs is PTA. With this method the Brownian motion of the EVs is analysed to yield both particle size and concentration data. The added benefit of the PTA method is that it provides details of the EV concentration in particles per millilitre, rather than as a protein concentration of micrograms per millilitre. As such, the concentration measurements obtained are less sensitive to the presence of contaminating protein in the sample, compared to standard protein-based quantification assays. It should be noted that PTA-based methods are not totally immune to the presence of contaminating proteins in the sample. Larger protein aggregates may still influence the concentration measurements, as they will also be within the typical size range of EVs. Further refinements to the process to include the use of fluorescently labelled EVs may also allow for easier identification and elimination of protein aggregates that may hinder accurate EV concentration measurements [45].

A key assay that has been developed with respect to the study of antigen and antibody interactions of EVs is the Enzyme Linked Immunosorbent Assay (ELISA). ELISA can detect EVs using antibodies specific to surface markers, such as CD9 and can be utilised with human blood plasma samples and cell culture media. Its ability to be effective using clinical samples highlight its importance as an assay suitable for use in precision medicine. However, subsequent assays have been developed such as ExoScreen, which have demonstrated a broader working range than ELISA and additionally have minimal sample handling involved [19]. It has not been directly stated but it has been suggested, that the pre-analytical conditions that can affect the small EVs utilised in a microarray protocol could in fact affect the phenotype of small EVs used in other immunoaffinity methods, such as ELISA [28]. These are challenges that must be assessed and overcome to fully enable the use of EVs in precision medicine-based approaches.

Flow cytometry is a fluorescent labelling method that can also be used for EV characterisation. Through use of dyes specific to EV components (including proteins, RNAs \& lipids), EVs can be characterised from a sample. A caveat to be considered with this approach is that there may be a background signal present in the sample, caused by the presence of unconjugated fluorescent label. Recently, a 
specialised protocol using Nano-FACS, along with SEC to remove the unconjugated label was devised. This was done to minimise the extent of unconjugated label present in a sample [49].

Recent work by researchers in Miltenyi Biotec GmbH (Germany) show recent developments in affinity-based binding approaches for EV isolation and characterisation. They have developed a multiplex polystyrene bead platform which can be used to detect up to 39 surface markers present in a single sample and can allow for visualisation of them using antibodies. These distinct EV subpopulations can be characterised using flow cytometry. They have additionally applied a high-resolution microscopy detection method called Stimulated Emission Depletion (STED) which can be used to look at individual EVs. STED can be used now to detect the presence of more than one fluorescent antibody on an EV [50].

The characteristic proteins that occur as surface markers along with their cargoes are compiled in a variety of annotated databases that are frequently updated with recent publications. There are the broader EV databases such as EVPedia [7] and VesiclePedia [8], and there is also a more exosome specific Exocarta database [6]. It can be found upon examining these databases that EV contents are not simply limited to proteins, but also can contain mRNAs, miRNAs and lipid molecules.

\section{EV Contents}

Initially, EVs were reported to contain proteins, yet it has been subsequently shown that they can also contain RNA and DNA species, which can impact downstream gene expression. The levels of DNA content are very low in comparison to the level of RNA content present. The levels of the different RNA subtypes (especially ribosomal RNA [rRNA]) can differ [5]. The EV RNAs that are trafficked to target cells are referred to as EV shuttle RNAs (esRNAs). esRNAs were shown to be chiefly small non-coding RNAs, such as miRNAs. Upon closer examination of mast cell EVs, it was reported that a fraction of their RNA content was parentally shared and that there were several EV specific RNA transcripts found. There were a number of miRNAs found in the mast cell EVs that have been implicated in processes such as tumorigenesis, angiogenesis and exocytosis [51]. Subsequent roles for certain EV's RNA content in differentiation [52] and cellular repair have been reported. These effects have been shown in the liver [12] and in bone cells [53]. These EVs could act as a form of cellular communication, through which the shuttling of EV contents from one cell to another may moderate processes in target cells. It has been shown that the RNA content of EVs are stable and can be protected while they are being shuttled in the extracellular environment, even in RNAse presence [5]. It has been reported that secreted EVs can carry a significant proportion of origin cell mRNA content and miRNAs as cargo [54].

It has been shown that the $\mathrm{N}$-linked glycosylation of certain subgroups of EV proteins and can affect the extent of their packing into the EV or association with the EV surface by colocalisation. An example of a typical EV glycoprotein and a non-glycosylated EV protein are the CD63 and CD81 tetraspanin proteins. These are EV membrane associated by colocalisation. In cases of low numbers of glycosylated sites, low EV recruitment of EW1-2 (EV glycoprotein) is observed. If there are high numbers of glycosylation sites present, there is a higher degree of EW1-2 recruitment to the EVs [55].

EV contents can be representative of the nature of their cell of origin, particularly in the case of cancer tissues. It has been shown that certain primary tumour proteins are shared by the EVs they produce. For example, EVs produced by Non-Small Cell Lung Cancer (NSCLC) primary tumours share high levels of EpCAM and Insulin Growth Factor 1 Receptor ( $\alpha$-IGF1R). EVs from ovarian cancers share high levels of EpCAM and CA125 with the primary tumour tissue also [18]. Additionally, it has been shown that Epidermal Growth Factor Receptor (EGFR) is a marker for NSCLC [56] and EGFR is also possessed by EVs derived from ovarian cancer cells [57]. Variants of EGFR such as EGFRvIII can be a cargo of glioblastoma derived EVs [10]. EVs that are secreted by stem cells can carry Vascular Endothelial Growth Factor (VEGF) as cargo, which may facilitate their regenerative effects [15] and they have been implicated in 
affecting the EGFR signalling pathway additionally [58].

It was initially shown, that melanoma cells could produce subpopulations of EVs, which can be classified as high-density EVs and low-density EVs. This classification considers differences in size, high-density EVs peaking at $\sim 78 \mathrm{~nm}$ on average and the low-density EVs peaking at $\sim 117$ nm. Using other cells lines, it was shown that these EV subpopulations were not melanoma specific and they possess their own respective protein composition, mRNA cargoes and effects on downstream gene expression. The highdensity EVs displayed a lack of rRNA and showed a broader range of small RNA sizes, as compared to the low-density EVs. There are differences in the enrichment of specific protein classes between the EV subpopulations. Highdensity EVs show high levels of ribonucleoproteins and ribosomal proteins, whereas low-density EVs have higher levels of G protein modulators and a slight increase in dehydrogenase levels [17].

EV miRNA cargo can be subdivided in $n$ miRNA (neutral carried miRNA) and s-miRNA (selectively released miRNA). A difference between these miRNA subclasses is that $\mathrm{n}$ miRNAs are carried by unconventional EVs, which are slightly larger than normal EVs and the s-miRNAs are carried by more typical EVs. These unconventional EVs and conventional EVs differ by the presence of elevated CD44 in the case of the unconventional EVs. The release of nmiRNA carrying EVs is unaffected by malignancy; however, the release of EVs carrying s-miRNA is increased in cases of malignancy. This highlights how the state of the cell can alter EV release and the cargo of miRNAs that are carried by the EVs [59]. This list is by no means exhaustive, it merely provides certain examples. The roles of miRNAs in cell biology research is under constant development.

Through their diverse contents, EVs can affect target cells in many ways, both positively and negatively. EVs are known to act in the transfer of genetic material and proteins. Here, using examples and depicted in Figure 2, EVs can be shown to play roles in pathological and regenerative processes in the cell with applications in precision and personalised medicine.

\section{Pathological Roles of EVs}

EVs can act as mediators of extracellular environment remodelling and signal transduction. Tumour EVs have been shown to induce cellular differentiation from fibroblasts to myofibroblasts. Myofibroblasts, while transiently present, have been reported to play a role in healthy tissue repair. Yet, if they are persistently present, they can play a role in organ fibrosis, as well as promoting cancer progression through cancer cell environment alteration. It has been shown that specific cancer cell types produce EVs with Transforming Growth Factor- $\beta$ (TGF$\beta$ ) necessary to trigger this differentiation [52]. EVs trafficked from cancer cells can interact with target cells and confer certain properties that are cancer related. As an example, through transfer of tissue transglutaminase and crosslinked fibronectin from breast cancer cells to fibroblasts, cellular transformation can be induced [65]. Another process affected by EV interaction is host cell matrix modulation, which can increase a cancer cell's migratory potential. Tumour EVs have been shown to interact directly with the ECM by binding to the adhesion molecules and ECM components present, depending on the EV's adhesion molecules. Tumour EVs release proteases which can directly degrade ECM proteins, which modulates the target cell's ECM. This can act to increase the motility of stromal cells and the process of cellular migration, which can allow for increased wound healing observable, but can contribute to increased cancer cell invasiveness [62]. It has been reported that the EVs produced by cells undergoing an epithelial to mesenchymal transition possess a proteome with increased levels of proteases such as matrix metalloproteinase 14 (MMP14) and ADAM10 which can contribute to increased metastatic potential [66]. 


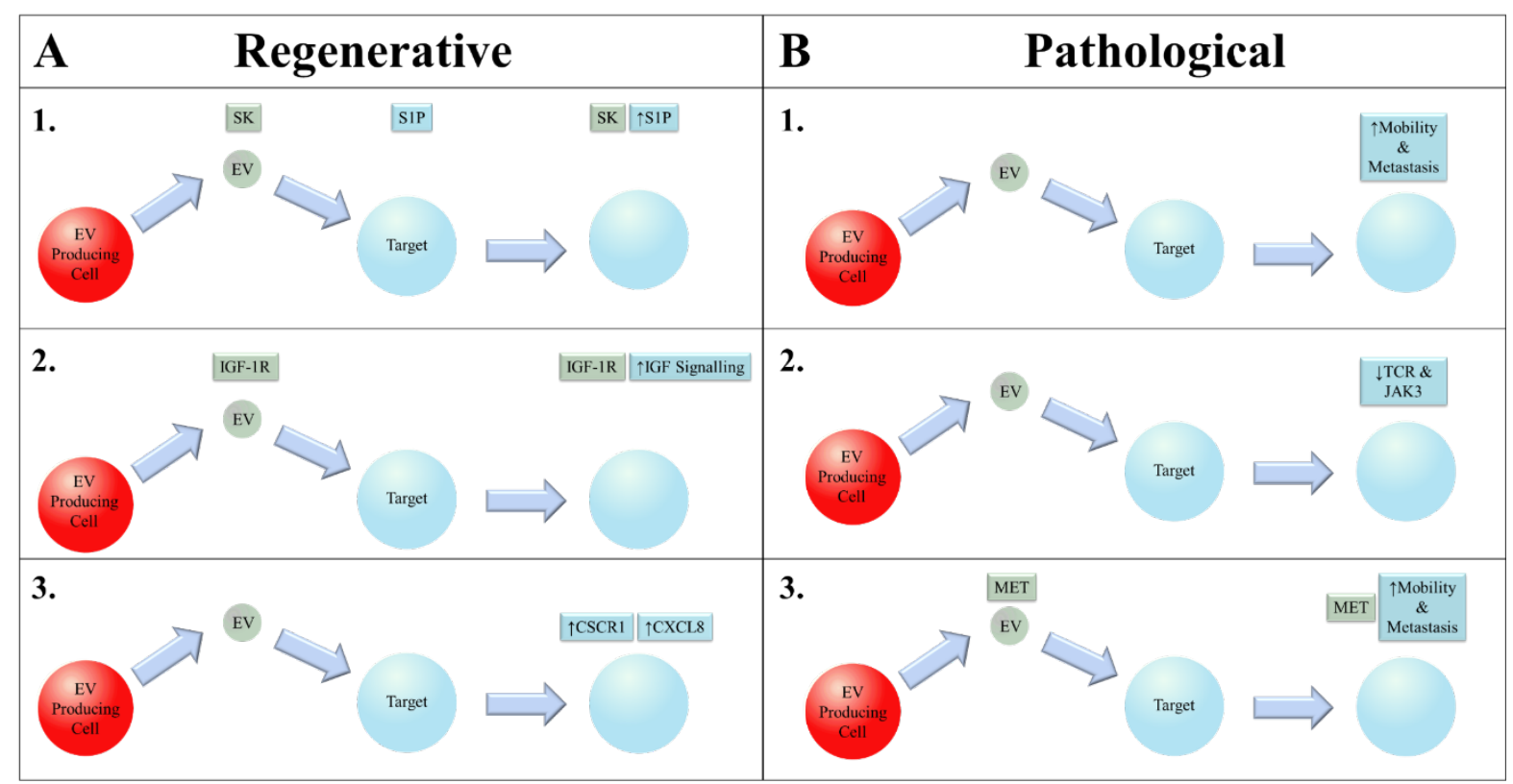

Figure 2. EVs can affect cells in regenerative (A) and pathological (B) manners which can impact downstream processes. (A) 1. EVs from hepatocytes can traffic Sphingosine Kinase (SK) to damaged hepatocytes, increasing Sphingosine-1-Phosphate (S1P) levels and increasing cellular proliferation [12]. 2. EVs can traffic IGF-1R mRNA to target cells, increasing IGF signalling and promoting regeneration of kidney tubule cells [60]. 3. EVs can increase secretion of cytokines that are implicated in regenerative processes such as CXCR1 and CXCL8 in target cells [61]. (B) 1. Tumour EVs can interact with a target cell and modulate their Extracellular Matrix (ECM), which can increase their mobility and metastatic potential [62]. 2. EVs can be implicit in the inhibition of T Cell Receptor (TCR) and JAK3 expression in target T cells which reduces the host's immune response [63]. 3. Melanoma tumour EVs can increase the mobility and metastatic potential of target cells through the horizontal transfer of MET oncoprotein [64].

EVs are implicated in signalling and communication to influence a cancer's Premetastatic Niche (PMN). Melanoma tumour $\mathrm{EVs}$ are found to interact with bone marrow derived cells and through horizontal transfer of MET oncoprotein can promote cellular mobilisation and increase metastatic potential of the cancer cells [64]. EVs are involved in an autocrine signalling process with Wnt11 to stimulate breast cancer cell mobilisation and metastasis [67]. EVs carrying migration inhibitory factor secreted by pancreatic cancer cells, can act to inhibit Kupffer cells and contribute to liver tissue-specific metastasis occurring [68]. It should be noted that the EVs alone do not facilitate the PMN formation, they act in conjunction with soluble elements of the adhesion matrix [69].

It has also been suggested that tumour EVs from Lewis lung carcinoma can play a role in Toll-Like Receptor 3 (TLR3) activation in lung epithelial cancer cells, which is implicated in the formation of the PMN. The tumour EVs are enriched with small nuclear RNA species, which may act as ligands for the TLR3 on the epithelial cells. The secretion of chemokines (for example, CXCL2 and CXCL5) is triggered upon TLR3ligand interaction and neutrophils are recruited by chemotaxis. The neutrophils upon recruitment, infiltrate the lung tissue and their presence can negatively affect patient prognosis [70].

It has been shown that tumour EVs can contain miRNAs (such as miR-210). These can potentially contribute to angiogenesis and downstream metastasis. Neutral Sphingomyelinase 2 (nSmase2) is involved in EV biogenesis and has been implicated in regulating the EV trafficking of miRNAs which can enhance the metastatic process. This has been shown using different cell lines [71]. EVs from melanoma cells have been shown to traffic to sentinel lymph nodes and induce a level of conditioning that can facilitate the occurrence of metastasis [72]. 
Immunomodulation as a process, especially upon EV intervention can be very complicated, as it can vary depending on the nature of the cell secreting EVs and the target immune cell. EVs can be implicated in immunosuppression upon release by acute myeloid leukaemia cells. These EVs can inhibit NKG2D expression by certain Natural Killer (NK) cells and reduce their downstream cytotoxicity. These EVs can also significantly reduce the levels of several immunereactive chemokines. They can increase the level of immunosuppressive adenosine produced by the target NK cells [9]. Tumour EVs can inhibit TCR and JAK3 expression, while inducing T cell apoptosis additionally [63]. Immune surveillance can be triggered by EVs in certain cases. Through high levels of Pigment Epithelium Derived Factor (PEDF) present on the EVs produced by nonmetastatic melanoma cells, they can be taken up by myeloid progenitors in the bone marrow. This can improve the host's immune response to the cancer. Upon EV interaction, the myeloid progenitors in the bone marrow can induce and increase the levels of patrolling NK cells at the PMN, which can phagocytose and kill metastatic melanoma cells [73]. This is an example of a naturally occurring immunotherapy for melanoma. It was also shown that EVs secreted by primary melanoma cells can suppress lung metastasis. Based on this example, it is thought that through use of PEDF derivative peptides successfully loaded onto EVs, that EVs could be utilised as a molecular means of suppressing cancer metastasis in certain cases [73].

EVs secreted from endothelial cells can act as a means of trafficking anti-inflammatory signals (through miRNA content) to target cells. This can involve inhibition of Nuclear Factor light chain Kappa activated enhancer of B cells (NF- $\kappa B$ ) in the monocytes which can decrease their activation. However, EVs has also been suggested to play a role in promoting inflammation in pericytes and may be implicated in mediating neovascularisation occurring [74]. Altered EV function can be a factor implicated in cardiovascular disease [75]. The role of secreted EVs have also been reported to include the induction of inflammatory cell adhesion through ICAM-1 trafficking. EVs in this case can promote the progression of atherosclerotic plaque development [76]. The pathological role of EVs in relation to inflammation, particularly in cardiovascular disease is quite complex and requires more comprehensive elucidation.

EVs can also be affected by the therapeutic processes that the patient has undergone. EVs produced by radiotherapy exposed cancer cells can induce a metastatic phenotype in local cancer cells through paracrine signalling. Tumour EVs were shown to induce expression of the AKT signalling pathway (through phosphorylated mTOR) and increase chemotactic cellular motility. AKT signalling results in increased MMP expression in cancer cells, which can increase invasiveness and metastatic potential. It has been suggested that inhibitors of AKT and mTOR could decrease the level of metastasis that occurs after ionising radiation exposure. It has also been reported that preclinical testing following this rationale has shown promising results. Exact determination of the key metastasis inducing proteins is needed for EVs to be potentially used in molecular therapies [77]. It has also been reported that the level of EV secretion from glioblastoma cells and astrocytes can increase upon radiation exposure. The EV population released by glioblastoma cells can induce increased IGFBP2, p-TrkA, p-FAK levels in recipient cells and can increase cell migration especially over EVs produced by non-irradiated cells [78]. EVs can also be released from bystander cells in cases of contact by radiation induced ultraviolet biophotons. These EVs were subsequently shown to decrease recipient cell survival upon incubation. However, RNAse contact with the EVs prior to their incubation with the recipient cells reduced this decreased survival effect [79]. EVs clearly can have a myriad of roles in radiation response which merits further investigation. EVs are involved in multiple physiological processes and this allows for their potential use in a multitude of therapeutic applications.

\section{Therapeutic Roles of EVs}

EVs can be implicated in modulating the secretome of a target cell (e.g., a Peripheral Blood Mononuclear Cell [PBMC]). The target cell, upon EV interaction can have altered gene expression in proangiogenic and repair pathways. The cells also demonstrate increased secretion of 
glycolipids that are involved in the regulation of angiogenic processes and inflammation response. These EVs can also induce the secretion of cytokines, such as CXCR1 and CXCL8 in target cells, which are implicated in regenerative processes [61].

Regeneration across multiple cell types can be positively influenced and mediated by EV interaction with a target cell. A further example of this, is the EV mediated horizontal transfer of the IGF-1R mRNA from a Mesenchymal Stem Cell (MSC) to a proximal tubule cell found in the kidney. This allows for IGF pathway mediated proliferation and regeneration of cells in the damaged tissue to occur [60]. Additional examples of EVs' role in initiating and promoting regeneration can be found in multiple tissues. These are mediated by processes such as AKT signal pathway upregulation in cardiomyocytes [15] and NF- $\mathrm{KB}$ activation promoting angiogenesis in endothelial cells [58]. These examples and their potential for clinical application will be discussed further below.

\section{Regenerative roles of EVs}

EVs that are derived from stem cell tissues have been implicated as a means of triggering regeneration of certain cell types. As an example, EVs derived from MSCs can trigger regeneration of bone cartilage tissue. The cells regenerated using EV treatment showed good levels of integration into the existing host cartilage tissues and strong regeneration of the subchondral bone. This highlights the efficacy of stem cell EV mediated action in tissue repair. It should be noted that the exact mechanism underlying this process has not been elucidated, though it is suspected to be due to EV cargo [53].

Interestingly, it has been shown that EVs derived from hepatocytes can also play a role in tissue proliferation and regeneration in target hepatocytes. Through trafficking of specific cargo that can affect target cell signal transduction, EVs can trigger proliferative signals. This was reported to occur by the transfer of SK2 carried in EVs to target hepatocytes, which acts to increase S1P production. This in turn, promoted hepatocyte proliferation both in vitro and in vivo. It was also shown that the other liver associated cells did not produce EVs that positively affected the regeneration of hepatocytes [12]. If EVs loaded with ceramide and SK were successfully targeted to other hepatocytes through the utilisation of a hepatocyte marker protein, then perhaps this regeneration mechanism could be induced in the liver synthetically. This could allow for increased therapeutic options in cases of disease treatment and chronic condition management [12].

Regeneration of the peripheral nervous system can involve EVs. EVs secreted by Schwann cells are implicated in axonal regeneration in the peripheral nervous system. It was shown that Schwann cell EVs were effective at axonal regeneration, while fibroblast EVs were ineffective. It is thought that EVs inhibit the RhoA GTPase activity in the target cell and modify neuron growth cone dynamics to promote elongation. These EVs have shown efficacy in stimulating axonal regeneration both in vitro and in vivo. Further investigation is required to elucidate which of the EV contents are facilitating this axonal regeneration process, as it may offer potential for regeneration to be induced in other parts of the nervous system [13].

Post myocardial infarction, EVs have been shown to be secreted by MSCs and can have a protective effect on cardiomyocytes. These EVs are enriched in CXCR4, which demonstrate increased anti-apoptotic effects compared to EVs that are low in CXCR4. There are increased levels of phosphorylated AKT, IGF-1 $\alpha$ and procaspase 3 present in these EVs and the level of caspase 3 is decreased. This occurs by the IGF-1 $\alpha$ inhibiting the cleavage of procaspase 3 to caspase 3. These EVs also display upregulated VEGF signalling and have the potential to initiate angiogenesis in vitro. Upon in vivo testing, the CXCR4 enriched EVs demonstrate increased levels of angiogenesis and reduced infarction size. EVs have been highlighted as a promising new method of myocardial injury treatment, yet there are refinements needed in stability, integrity, and potency to be conducted. It is thought if CXCR4 expression could be increased in the MSCs, secretion of EVs containing angiogenic factors could be increased also [15]. Additionally, it has been reported that specific EV miRNA cargo (miR-210 and miR-132) have anti-apoptotic and proangiogenic roles in relation to cardiomyocytes. These EV stimulated 
activities are linked with decreased severity in cardiac tissue damage post myocardial infarction [80]. EVs are also thought to promote cardio protection through interaction of surface Heat Shock Protein 70 (HSP70) with target cell TLR4 which induces ERK signalling pathway and the downstream phosphorylation of HSP27, which induces cardio protection [81].

Cardiac progenitor cells have been found to secrete EVs in hypoxic conditions. It is shown here, that environmental conditions can alter the EVs being produced by a cell and their cargo. Hypoxic Cardiac Progenitor Cell (HCPC) derived EVs were shown to be more effective in stimulating endothelial cell mediated tubule regeneration, than EVs produced in oxygen normative conditions. It was additionally shown that the regeneration observed is dependent on the miRNA cargo. HCPC derived EVs were shown to have a reducing effect on profibrotic genes in fibroblast cells, as compared to EVs produced in oxygen normative conditions. There were 11 miRNAs found to be upregulated in hypoxic conditions and the EVs produced by HCPCs possessed seven of these miRNAs respectively. The EVs were also shown to affect certain miRNA expression in target cells. These HCPC produced EVs were applied to an in vivo model of ischaemic reperfusion injury to assess their efficacy in clinical treatment. Only a subset of EVs produced from cells exposed to hypoxia for 12 hours showed positive response and increased function of the tissue, while also significantly reducing the extent of fibrosis that occurred [16].

EVs' regenerative capability has been shown to promote the differentiation of some cell types through paracrine cell signalling. Upon differentiation of human skeletal myoblasts to myotubes, EVs secreted are thought to play a role in inducing and promoting the differentiation of Human Adipose-derived Stem Cells (HASCs). The EVs produced by human skeletal myoblasts were shown to have increased levels of proteins and growth factors associated with regulating the regeneration and development of skeletal muscle. Examples of these proteins include, VEGF, VEGFR3, Insulin Growth Factor, IGF Binding Protein-3 (IGFBP3), TGF- $\beta$ 1,3 and Platelet Derived Growth Factor (PDGF)-AA. EVs produced by differentiating myoblasts have been shown to accelerate the differentiation of HASCs. These EVs have been shown to reduce fibrosis and increase regeneration of the muscle tissue, compared to non-EV treated controls in an in vivo context. However, application of excess amounts of EVs may lead to deleterious effects, including apoptosis [14].

MSC derived EVs promote angiogenesis and contribute to activation of $\mathrm{NF}-\kappa \mathrm{B}$ in endothelial cells. In certain disease states that can induce a hypoxic environment, there is increased secretion of proangiogenic factors and EVs by MSCs. Proteomic analysis of MSCs shows the expression of the lipid biosynthesis and angiogenic pathways are increased in hypoxic conditions. This increase in lipid biosynthesis could facilitate the increased levels of MSC EV secretion observable in hypoxic conditions or could be an adaptive response. The EVs were shown to promote angiogenesis through the NF$\kappa \mathrm{B}$ pathway, as upon addition of an NF- $\kappa \mathrm{B}$ inhibitor (PDTC), angiogenesis was inhibited. The EVs are enriched in EGFR, FGF and PDGF Receptor signalling pathway molecules. Yet, it is unclear whether the angiogenesis induced is the result of direct action on the endothelial cells or indirect action through immune cells, as there may be other signalling contributors involved. It has also been shown that EVs act in a dosedependent manner and there may be a limit for positive effect produced [58].

EVs secreted by endothelial progenitor cells have been implicated in increasing the vascularisation of pancreatic islets in an in vivo model. This highlights the capability of EVs in driving angiogenesis in tissues in a regenerative context. This allows for sustained insulin secretion by these pancreatic islet cells [82]. Additionally, EVs derived from adipose MSCs that are stimulated with PDGF possess c-kit and CSF. With this cargo, trafficked EVs display increased proangiogenic capabilities in target cells, yet inhibition of these molecules also decreases the EVs' proangiogenic capabilities [83].

EVs capability to induce angiogenesis, varied cargoes, and carrying ability provides the opportunity for modified transport applications. 
Utilisation of EVs as drug delivery vehicles

Molecular based therapies and drug therapies can involve EVs being used as delivery vehicles. In cancer therapy, a major issue is the cancer can develop multiple drug resistance. This can be due to expression of the MDR1 gene and expression of drug efflux membrane transporters. Macrophage derived EVs can be successfully utilised as a drug (e.g. paclitaxel) vector into cancer cells. These EVs can accumulate in resistant cancer cells and result in greater cytotoxicity occurring, as compared to conventional administration. Additionally, those cells that overexpress the drug efflux transporter show higher cytotoxicity upon EV treatment, than those that do not. The EV carried drug can more effectively circumvent drug resistance mechanisms found in the target cells, through endocytosis and other mechanisms. There are still several improvements to be made before methods such as this can be applied in a precision medicine context. However, promising efficacy has been shown in an in vivo context for their use in treatment of cancers presenting with pulmonary metastases [25]. Packaging of cytotoxic drugs in EVs can improve drug delivery efficacy over normal drug in an in vitro context. As an example, this method has been reported to inhibit ovarian cancer cell mediated drug efflux and therefore decrease ovarian cancer cell chemoresistance [84].

EVs can be utilised as vehicles for the precise transfer of siRNAs into target cells, both in vitro and in vivo. EVs utilised derived from bone marrow dendritic cell have minimal immunogenic impact due to the reduced presence of immune stimulatory molecules. A caveat regarding this approach is that different cell lines can produce differing numbers of EVs and that the EV populations possess distinct characteristics. Once loaded with siRNAs, the EVs can be applied to cells in vitro and the siRNA amount incorporated can be adjusted. They can be successfully applied to in vivo models and their efficacy can be monitored and optimised. This method of delivery highlights the potential for engineering EVs as vectors for miRNAs, miRNA mimetics and gene therapies [24].

EVs can be chemically transfected to contain siRNA molecules, which retain their functionality within the EVs and upon successful transfer to recipient cells. An example of this is the downregulation of RAD51 and can result in cell apoptosis occurring. This approach has yielded promising results in HeLa cell line testing in vitro[85]. An additional example of in vitro gene transfer using EVs involves the trafficking of siRNA to reduce the levels of $\alpha$-synuclein present in several cell lines. This testing was also conducted in vivo using mice and demonstrated the capability of reducing the level of pathological $\alpha$-synuclein [86].

siRNA carrying EVs can be directly injected into the brain, decreasing both the mRNA level and expression of protein. This method has shown efficacy in decreasing the expression of BACE1 (an Alzheimer's disease specific therapeutic target) in neurons, microglia and oligodendrocytes. The potential for EVs to be utilised in tissue-specific therapies is evident as they can be specifically targeted to cells based on the presence of specific markers (e.g., Lamp2b on EVs fused with neuron-specific peptide rabies virus glycoprotein (RVG). There is the potential issue of cellular toxicity that can be caused by siRNA being introduced to the cell, however this can be tested for using a cytotoxicity assay. The efficacy of an siRNA does not decrease across multiple consecutive applications. It can also induce less side effects and host immune responses than direct siRNA introduction, which may make this more amenable as a long-term therapy [87].

EVs for use in therapeutic interventions are already being patented and considered as commercially viable approaches. Orbsen Therapeutics (Ireland) as an example, have filed patents for the isolation and use of EVs containing Syndecan-2 (Sdc-2) [88]. This patent followed a patent previously filed for the use of Sdc-2 in clinical applications and perhaps will facilitate Sdc-2 EV mediated therapeutic research [89]. The interest in Sdc-2 could stem from the fact it is an adhesion molecule involved in motility and other processes that can be implicated in the increased pathogenesis of colorectal cancer [90].

Examples of the pathological and therapeutic aspects of EVs discussed above can be visualised in Figure 3. 
EVs also possess the capability for use as a diagnostic tool which is subsequently discussed.

\section{Diagnostic Uses of EVs}

As EVs can have manifold functions across multiple cell types, it has been suggested that their presence can be used as a diagnostic marker in certain disease states. EVs have been suggested as a means of cancer biomarker testing in a more non-invasive manner than the typical tissue biopsy approach. As a screening process, EV testing can allow for the detection of a cancer prior to presentation. This offers the opportunity for earlier diagnosis and better prognosis for patients which have highly lethal and late presenting cancers, such as pancreatic cancer [91] and NSCLC [92]. It is through examination of EV marker proteins and tumour specific marker proteins across different cancer tissues, that the merits of EV study for disease diagnosis become evident [18].

In previous research, EVs have shown to be present in a variety of bodily fluids including plasma and urine. They can be isolated, stained, and characterised using antibodies to marker proteins. There are many markers that can be used, including CD24. Bioanalyzers can be used to characterise specific SNPs in a CD24 EV mRNA that are implicated in disease risk, progression and can be associated with certain diseases, such as chronic hepatitis B and multiple sclerosis [93].

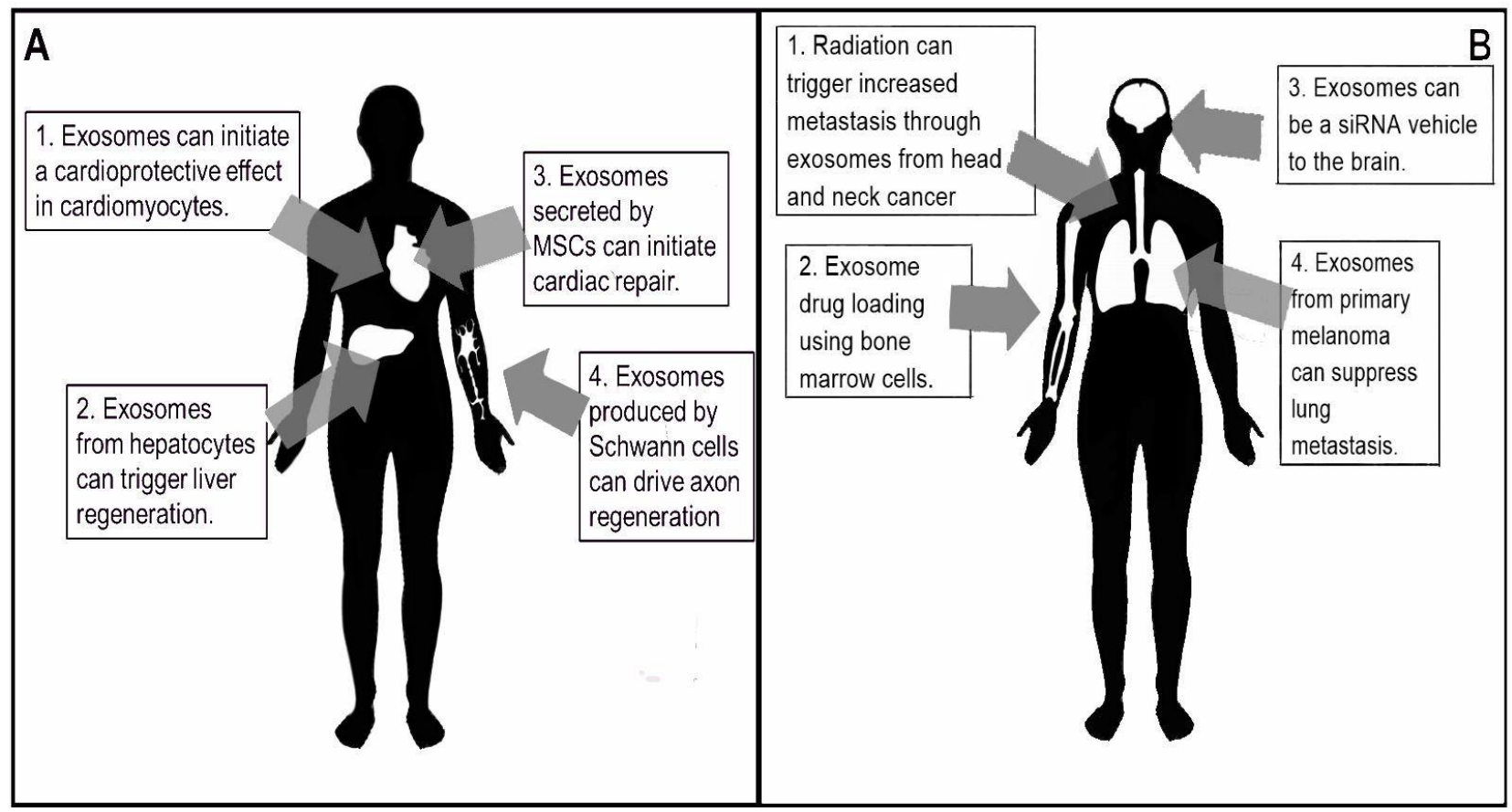

Figure 3. Diagram showing examples of EV sites of action in the human body in a (A) regenerative context and $(B)$ pathological context for precision medicine. (A) 1. EVs secreted by MSCs can have a cardioprotective role after injury through altered cardiomyocyte intracellular signalling [15]. 2. EVs secreted by hepatocytes can increase proliferation in damaged hepatocytes [12]. 3. Hypoxic cell produced EVS can influence target cells to initiate regeneration and reduce fibrosis occurring [16]. 4. EVS from Schwann cells can increase the regeneration of axons in the peripheral nervous system through alteration of growth dynamics [13]. (B) 1. After radiation therapy, EVs from head and neck cancer cells can increase the metastatic potential of target cells [77]. 2. EVS from bone marrow dendritic cells can be loaded successfully with paclitaxel and be used to target cells that possess drug resistance [25]. 3. EVs have shown potential for use as a tissue-specific siRNA vehicle in the treatment of neurodegenerative conditions [87]. 4. EVs from poorly metastatic melanoma cells can suppress lung metastasis occurring through bone marrow cell interaction, which increases the NK cells present at the PMN [73]. 
In urine, EVs can used as a direct diagnostic marker for prostate cancer. It has been highlighted that cancers can be heterogeneous in their mutation status and that an EV's transcriptome may be more informative of the overall progress of tumour than a biopsy. It has been shown that urine EVs can contain mRNA for Prostate Specific Antigen, which itself can be utilised as a prostate cancer biomarker. EVs can also contain the mRNA transcript of the fusion gene TMPRSS2:ERG, which is a diagnostic transcript for prostate cancer [94].

Based on the varied contents possessed by EVs, insight can be gained regarding cancer status. Upon investigation of pancreatic cancer EVs, it was shown many gene transcripts are present. A wide array of RNAs can be carried by the tumour EVs which are be implicated in processes such as apoptosis and transcription regulation. EV specific protein coding targets can also be uncovered and considered for downstream therapeutic targeting. A gene mining approach using existing databases can highlight which cancers express the highest number of EV genes, which may detail sets of EV genes associated with specific cancers. Considering the presence of specific EV miRNAs and lncRNAs, a panel of pancreatic cancer specific EV biomarkers could potentially be developed for diagnostic purposes. Also, it can display in which biological samples there are the highest numbers of EV transcripts, which could help direct potential diagnostic avenues to be investigated in the future [91].

Bioinformatic analysis of EVs found in differing biological samples (e.g., seminal plasma) can grant insight into tissue-specific cancers. It has been previously reported the protein composition of seminal plasma can vary

[96]. between individuals and is reflected in the EVs present. Bioinformatic investigations like this allow for examination of EV contents that are shared between individuals and those that are distinct. Seminal EV contents have been implicated in a variety of key biological processes including cell growth, maintenance and protein metabolism. Despite the variability between individuals, through comparison of healthy seminal EV content and disease state seminal EV content, there is potential for discovery of disease biomarkers for diagnosis [95].

esRNAs can be implicated in the level of chemotherapeutic resistance observed in cases of osteosarcoma. There is differential expression of the miRNAs carried by EVs in cases of high chemotherapeutic resistance and low chemotherapeutic resistance respectively. In poor response samples, several pathways are affected by the EV miRNA contents, including the Ras signalling pathway, the PI3K-AKT signalling pathway and the regulation of the pluripotency of stem cells. It has also been shown that certain mRNA contents are differentially expressed in good response and poor response cases respectively. A panel of eight miRNAs were identified as reliable biomarkers for poor chemotherapeutic response in osteosarcoma. These EV miRNAs (shown in Table 2) have been found to impact a variety of cellular processes, and their use in diagnosis and potential therapies have been postulated. EV protein and miRNA cargo have been shown to be utilized in the earlier diagnosis of typically late stage presenting NSCLC. Using an antibody-based assay, EVs can be characterized from plasma samples and used in lung cancer diagnosis.

Table 2. Examples of the respective miRNA cargoes that cancer derived EVs can carry.

\begin{tabular}{|c|c|c|c|c|c|c|c|c|}
\hline \multirow{2}{*}{$\begin{array}{l}\text { Cancer } \\
\text { Type } \\
\text { Pancreatic }\end{array}$} & \multicolumn{8}{|c|}{ miRNA cargo of cancer tissues } \\
\hline & Let-7a & $\operatorname{miR}-21$ & $\operatorname{miR}-23 \mathrm{a}$ & $\operatorname{miR}-150$ & $\begin{array}{c}\text { miR- } \\
223\end{array}$ & miR-1224-5p & $\operatorname{miR}-1229$ & $\begin{array}{l}\text { miR- } \\
1246\end{array}$ \\
\hline Colon & miR-9 & $\operatorname{miR}-27 \mathrm{a}$ & $\operatorname{miR}-124$ & miR-133a & $\begin{array}{l}\mathrm{miR}- \\
135 \mathrm{~b}\end{array}$ & miR-148a & $\begin{array}{c}\text { miR-199a- } \\
3 p\end{array}$ & miR-385 \\
\hline
\end{tabular}


Though individual marker proteins did not show substantial diagnostic value, differing combinations of markers allowed for varying diagnostic value. Based on the investigation conducted, it was shown that a combination of 30 different markers provided the highest accuracy. Interestingly, there was no observable increase in diagnostic value beyond 30 in terms of biomarkers used. There is further work to be conducted regarding the nature of biomarkers tested for in the study of specific cancer EVs. Perhaps through substitution of ill-suited markers with more optimal markers, overall efficacy could be increased. [92].

There are certain molecular markers associated with different cancers, such as Carcinoembryonic Antigen and Carbohydrate Antigen 19-9 (CA19-9) with colorectal cancer. These can be used in diagnosis, yet they lack sufficient sensitivity in early cancer stages. It has been shown that certain colon cancer EV miRNAs can be as diagnostic biomarkers. A panel of eight different EV miRNAs have significantly elevated levels in cancer cases compared to healthy controls, yet upon surgical treatment, the levels decreased. There are other miRNAs that have been found to be upregulated in colon cancer patients, yet those highlighted in Table 2 have shown good prognostic value in a clinical context [97].

There has been development of other diagnostic tools for specific cancers also. There is the ExoScreen method as an example. It boasts the capability to detect EVs from a biological sample such as serum in a fraction of the time, compared to the more conventional methodologies. It can be applied in a highthroughput manner to a variety of cancers. It can therefore be used as a sort of liquid biopsy for cancer. This has been shown to be applicable to colorectal cancer cases [19].

\section{Discussion}

Considerations Regarding Clinical Use of EVs

It has been suggested that the use of EVs as a cell-free therapy could be advantageous regarding adherence to regulatory standards and safety guidelines. Adherence to these is important as there are strict legal requirements regarding the use of biological materials therapeutically in humans. Additionally, EV isolation should adhere to Good Manufacturing Practice (GMP) [61]. Despite the promise of EVs as a potential therapy in different applications, there is considerable refinement required at many stages. Their functions can be varied as seen above and with each different function, different considerations must be accounted for. EVs can be classified as biological therapeutics, yet depending on their cargo, case specific guidelines should to be implemented for individual EV therapies, compared to standard biological therapies. Despite current clinical trials being conducted (e.g.,[ and [23]) there is still a great deal of development and standardisation to be conducted regarding all aspects of EV study, including isolation, characterisation, quality control and elucidation of the mode of action of EVs [98]. The TRAIN-EV project has been set up to address some of these issues with the goal of optimizing EV utilisation [99].

Perspective on the future of EVs in Precision Medicine

Based on the research reviewed above, it can be observed that there are limitations in EV isolation. Regarding size-based approaches, undesired materials of a similar size can be isolated additionally [32]. Considering affinitybased approaches, EV marker proteins can vary between EV populations. Through identification of recurrent marker proteins across tissue types [19], proteome analysis and comparative examination of additional tissue types this could be potentially resolved. Further investigation of lectin induced agglutination of EVs could be a potentially fruitful avenue for future investigation as it is an appealing approach [42].

There have been successful EV phase 1 clinical trials demonstrating $\mathrm{EV}$ function in beneficial immunomodulation in recurrent glioma patients [100] and their use as diagnostic markers in Parkinson's disease [101]. Ongoing phase 2 clinical trials for EVs include tissue repair post ischaemic stroke [102]. An additional trial involves EV profiling post radiotherapy in thyroid cancer as a potential prognostic marker [103]. These examples suggest that EVs can be utilised in regenerative, pathological, diagnostic 
and prognostic applications, and that they are not far off from tangible therapeutic benefit. In this reviewer's opinion, through these examples it has been shown that EVs can be applied in a variety of medical applications and have great future potential. However, the exact contents of EVs require greater investigation and scrutiny as they may allow for greater understanding of $\mathrm{EV}$ mechanism of action in their varied functions.

There is a pressing issue regarding the regulation of EVs as there is an ambiguity regarding even their exact definition. In certain clinical trials they are referred to as "biological" interventions [102], whereas they can also be classified as "other" forms of intervention [22]. As various EV applications developing in clinical trials have not received United States Food and Drug Administration approval for diagnostic tests [20] or regenerative medicine [102] a consensus needs to be reached regarding the exact status of EVs as a form of intervention.

\section{Conclusions}

EVs and their subsets are of great importance due to their wide range of biological roles, acting in both pathological and regenerative contexts. Similarly, in disease states, they can be utilised as a means of assessing disease diagnosis, prognosis, and treatment. EVs can moderate a vast variety of processes for the secreting cell and target cell, depending on the nature of the EV cargo. Further research on these cargoes, and the development of approaches to use EVs as drug delivery vehicles or medical devises will enable EVs to be utilised to their full potential in precision medicine. EVs have been reported in certain tissues to mediate cellular and tissue regeneration in vivo. There are examples above of how this phenomenon can be harnessed and applied for regenerative applications. Through correct manipulation, the healing of a variety of tissues is achievable in a research context, though there is still progress required before it can be successfully applied in a clinical context. EVs have also shown to be potent factors in tumorigenesis and metastasis for cancers. They can also interfere with the immune system and alter signal transduction. EVs can be utilised as vehicles to target disease gene expression through siRNA action and to traffic drugs to target multiple drug resistant cancer cells. Finally, EVs can be representative of their cell of origin, and thus can grant insight into the secreting cell's state, provided the cell-specific markers are present as part of the $\mathrm{EV}$. The presence of these markers and specific cargoes can be detected using a variety of assays and potentially allow a diagnosis to be made for a patient. EVs already show a great deal of promise in the clinic in pathological, regenerative, and diagnostic contexts in precision medicine. With this in mind, the field of EVs and their future applications is certainly an area to watch.

\section{Acknowledgements}

The authors would like to thank the Master in Molecular Medicine programme under the School of Medicine at Trinity College Dublin for the encouragement and the partial financial support. The authors would also like to thank Science Foundation Ireland for the partial financial support under the AMBER centre under Grant Number SFI/12/RC/2278. Special thanks to Ms. Julia Malinowska for her contribution and revision of Figures $2 \& 3$.

\section{Conflict of interest}

The authors have no relevant financial or non-financial interests to disclose. For signed statements contact the journal office editor@precisionnanomedicine.com.

Quote this article as Savage, J, Maguire, CM, Prina-Mello A. Origins to Outcomes: A Role for Extracellular Vesicles in Precision Medicine. Precis. Nanomed. 2018, Apr; 1(1):18-41. 


\section{References}

1. Mora, E.M., S. Álvarez-Cubela, and E. Oltra, Biobanking of Exosomes in the Era of Precision Medicine: Are We There Yet? International Journal of Molecular Sciences, 2016. 17(1): p. 13.

2. Hessvik, N.P. and A. Llorente, Current knowledge on exosome biogenesis and release. Cellular and Molecular Life Sciences, 2017. 75(2): p. 193-208.

3. Harding, C., J. Heuser, and P. Stahl, Endocytosis and intracellular processing of transferrin and colloidal gold-transferrin in rat reticulocytes: demonstration of a pathway for receptor shedding. European journal of cell biology, 1984. 35(2): p. 256-263.

4. Johnstone, R.M., et al., Vesicle formation during reticulocyte maturation. Association of plasma membrane activities with released vesicles (exosomes). J Biol Chem, 1987. 262(19): p. 9412-20.

5. Palanisamy, V., et al., Nanostructural and Transcriptomic Analyses of Human Saliva Derived Exosomes. PLOS ONE, 2010. 5(1): p. e8577.

6. Keerthikumar, S., et al., ExoCarta: A web-based compendium of exosomal cargo. Journal of molecular biology, 2016. 428(4): p. 688-692.

7. $\quad$ Kim, D.-K., et al., EVpedia: an integrated database of high-throughput data for systemic analyses of extracellular vesicles. Journal of Extracellular Vesicles, 2013. 2(1): p. 20384.

8. Kalra, H., et al., Vesiclepedia: A Compendium for Extracellular Vesicles with Continuous Community Annotation. PLOS Biology, 2012. 10(12): p. e1001450.

9. Hong, C.S., et al., Circulating exosomes carrying an immunosuppressive cargo interfere with cellular immunotherapy in acute myeloid leukemia. Sci Rep, 2017. 7(1): p. 14684.

10. Skog, J., et al., Glioblastoma microvesicles transport RNA and protein that promote tumor growth and provide diagnostic biomarkers. Nature cell biology, 2008. 10(12): p. 1470-1476.

11. Tanaka, Y., et al., Clinical impact of serum exosomal microRNA-21 as a clinical biomarker in human esophageal squamous cell carcinoma. Cancer, 2013. 119(6): p. 1159-1167.

12. Nojima, H., et al., Hepatocyte exosomes mediate liver repair and regeneration via sphingosine-1phosphate. Journal of Hepatology, 2016. 64(1): p. 60-68.

13. Lopez-Verrilli, M.A., F. Picou, and F.A. Court, Schwann cell-derived exosomes enhance axonal regeneration in the peripheral nervous system. Glia, 2013. 61(11): p. 1795-1806.

14. Choi, J.S., et al., Exosomes from differentiating human skeletal muscle cells trigger myogenesis of stem cells and provide biochemical cues for skeletal muscle regeneration. Journal of Controlled Release, 2016. 222(Supplement C): p. 107-115.

15. Kang, K., et al., Exosomes Secreted from CXCR4 Overexpressing Mesenchymal Stem Cells Promote Cardioprotection via Akt Signaling Pathway following Myocardial Infarction. Stem Cells International, 2015. 2015: p. 14.

16. Gray, W.D., et al., Identification of Therapeutic Covariant MicroRNA Clusters in HypoxiaTreated Cardiac Progenitor Cell Exosomes Using Systems Biology. Circulation Research, 2015. 116(2): p. 255-263.

17. Willms, E., et al., Cells release subpopulations of exosomes with distinct molecular and biological properties. Scientific Reports, 2016. 6: p. 22519.

18. He, M., et al., Integrated immunoisolation and protein analysis of circulating exosomes using microfluidic technology. Lab on a Chip, 2014. 14(19): p. 3773-3780.

19. Yoshioka, Y., et al., Ultra-sensitive liquid biopsy of circulating extracellular vesicles using ExoScreen. Nature Communications, 2014. 5: p. 3591.

20. Tun, R. and R.F. Tutrone, Decision Impact Trial of the ExoDx Prostate (IntelliScore). Retrieved from https://clinicaltrials.gov/ (Identification No. NCT03235687). 2017.

21. Shen, Y. and W. Zhang, Circulating Exosome RNA in Lung Metastases of Primary High-Grade Osteosarcoma. Retrieved from https://clinicaltrials.gov/ (Identification No. NCT03108677). 2017.

22. Masatoshi, J., Effect of Plasma Derived Exosomes on Cutaneous Wound Healing. Retrieved from https://clinicaltrials.gov/ (Identification No. NCT02565264). 2015. 
23. Luginbuhl, A., Metformin Hydrochloride in Affecting Cytokines and Exosomes in Patients With Head and Neck Cancer. Retrieved from https://clinicaltrials.gov (Identification No. NCT03109873). 2017.

24. El-Andaloussi, S., et al., Exosome-mediated delivery of siRNA in vitro and in vivo. Nature Protocols, 2012. 7: p. 2112.

25. Kim, M.S., et al., Development of exosome-encapsulated paclitaxel to overcome MDR in cancer cells. Nanomedicine, 2016. 12(3): p. 655-664.

26. Lee, Y., S. El Andaloussi, and M.J. Wood, Exosomes and microvesicles: extracellular vesicles for genetic information transfer and gene therapy. Hum Mol Genet, 2012. 21(R1): p. R125-34.

27. Akers, J.C., et al., Biogenesis of extracellular vesicles (EV): exosomes, microvesicles, retroviruslike vesicles, and apoptotic bodies. Journal of Neuro-Oncology, 2013. 113(1): p. 1-11.

28. Bæk, R., et al., The impact of various preanalytical treatments on the phenotype of small extracellular vesicles in blood analyzed by protein microarray. Journal of Immunological Methods, 2016. 438(Supplement C): p. 11-20.

29. Lobb, R.J., et al., Optimized exosome isolation protocol for cell culture supernatant and human plasma. Journal of Extracellular Vesicles, 2015. 4(1): p. 27031.

30. Livshits, M.A., et al., Isolation of exosomes by differential centrifugation: Theoretical analysis of a commonly used protocol. Scientific Reports, 2015. 5: p. 17319.

31. Li, P., et al., Progress in Exosome Isolation Techniques. Theranostics, 2017. 7(3): p. 789-804.

32. Böing, A.N., et al., Single-step isolation of extracellular vesicles by size-exclusion chromatography. Journal of Extracellular Vesicles, 2014. 3(1): p. 23430.

33. Lee, K., et al., Acoustic purification of extracellular microvesicles. ACS Nano, 2015. 9(3): p. 2321-7.

34. Wu, M., et al., Isolation of exosomes from whole blood by integrating acoustics and microfluidics. Proceedings of the National Academy of Sciences, 2017. 114(40): p. 10584-10589.

35. Rider, M.A., S.N. Hurwitz, and D.G. Meckes Jr, ExtraPEG: A Polyethylene Glycol-Based Method for Enrichment of Extracellular Vesicles. Scientific Reports, 2016. 6: p. 23978.

36. Weng, Y., et al., Effective isolation of exosomes with polyethylene glycol from cell culture supernatant for in-depth proteome profiling. Analyst, 2016. 141(15): p. 4640-4646.

37. He, M. and Y. Zeng, Microfluidic Exosome Analysis toward Liquid Biopsy for Cancer. Journal of Laboratory Automation, 2016. 21(4): p. 599-608.

38. Fang, S., et al., Clinical application of a microfluidic chip for immunocapture and quantification of circulating exosomes to assist breast cancer diagnosis and molecular classification. PLOS ONE, 2017. 12(4): p. e0175050.

39. Pedersen, K., et al., Direct Isolation of Exosomes from Cell Culture: Simplifying Methods for Exosome Enrichment and Analysis. Translational Biomedicine, 2015. 6: p. 1-9.

40. Yoshioka, Y., et al., Comparative marker analysis of extracellular vesicles in different human cancer types. Journal of Extracellular Vesicles, 2013. 2(1): p. 20424.

41. Durcin, M., et al., Characterisation of adipocyte-derived extracellular vesicle subtypes identifies distinct protein and lipid signatures for large and small extracellular vesicles. Journal of Extracellular Vesicles, 2017. 6(1): p. 1305677.

42. Gerlach, J.Q., et al., Urinary nanovesicles captured by lectins or antibodies demonstrate variations in size and surface glycosylation profile. Nanomedicine, 2017. 12(11): p. 1217-1229.

43. Samsonov, R., et al., Lectin-induced agglutination method of urinary exosomes isolation followed by mi-RNA analysis: Application for prostate cancer diagnostic. The Prostate, 2016. 76(1): p. 68-79.

44. Yoshioka, Y., et al., Comparative marker analysis of extracellular vesicles in different human cancer types. Journal of Extracellular Vesicles, 2013. 2: p. 10.3402/jev.v2i0.20424.

45. Dragovic, R.A., et al., Sizing and phenotyping of cellular vesicles using Nanoparticle Tracking Analysis. Nanomedicine, 2011. 7(6): p. 780-788.

46. Conde-Vancells, J., et al., Characterization and Comprehensive Proteome Profiling of Exosomes Secreted by Hepatocytes. Journal of proteome research, 2008. 7(12): p. 5157-5166. 
47. Wang, J., et al., Identification and analysis of exosomes secreted from macrophages extracted by different methods. International Journal of Clinical and Experimental Pathology, 2015. 8(6): p. 61356142.

48. Sunkara, V., H.K. Woo, and Y.K. Cho, Emerging techniques in the isolation and characterization of extracellular vesicles and their roles in cancer diagnostics and prognostics. Analyst, 2016. 141(2): p. 371-81.

49. $\quad$ Morales-Kastresana, A., et al., Labeling Extracellular Vesicles for Nanoscale Flow Cytometry. Scientific Reports, 2017. 7(1): p. 1878.

50. Koliha, N., et al., A novel multiplex bead-based platform highlights the diversity of extracellular vesicles. Journal of Extracellular Vesicles, 2016. 5: p. 10.3402/jev.v5.29975.

51. Valadi, H., et al., Exosome-mediated transfer of mRNAs and microRNAs is a novel mechanism of genetic exchange between cells. Nat Cell Biol, 2007. 9(6): p. 654-9.

52. Webber, J., et al., Cancer Exosomes Trigger Fibroblast to Myofibroblast Differentiation. Cancer Research, 2010. 70(23): p. 9621-9630.

53. Zhang, S., et al., Exosomes derived from human embryonic mesenchymal stem cells promote osteochondral regeneration. Osteoarthritis and Cartilage, 2016. 24(12): p. 2135-2140.

54. Ekström, K., et al., Characterization of $m R N A$ and microRNA in human mast cell-derived exosomes and their transfer to other mast cells and blood CD34 progenitor cells. Journal of Extracellular Vesicles, 2012. 1(1): p. 18389.

55. Liang, Y., et al., Complex N-Linked Glycans Serve as a Determinant for Exosome/Microvesicle Cargo Recruitment. The Journal of Biological Chemistry, 2014. 289(47): p. 32526-32537.

56. Sandfeld-Paulsen, B., et al., Exosomal proteins as prognostic biomarkers in non-small cell lung cancer. Molecular Oncology, 2016. 10(10): p. 1595-1602.

57. Liang, B., et al., Characterization and proteomic analysis of ovarian cancer-derived exosomes. Journal of Proteomics, 2013. 80(Supplement C): p. 171-182.

58. Anderson, J.D., et al., Comprehensive Proteomic Analysis of Mesenchymal Stem Cell Exosomes Reveals Modulation of Angiogenesis via Nuclear Factor-KappaB Signaling. STEM CELLS, 2016. 34(3): p. 601-613.

59. Palma, J., et al., MicroRNAs are exported from malignant cells in customized particles. Nucleic Acids Research, 2012. 40(18): p. 9125-9138.

60. Tomasoni, S., et al., Transfer of Growth Factor Receptor mRNA Via Exosomes Unravels the Regenerative Effect of Mesenchymal Stem Cells. Stem Cells and Development, 2013. 22(5): p. 772-780. 61. Beer, L., et al., Analysis of the Secretome of Apoptotic Peripheral Blood Mononuclear Cells: Impact of Released Proteins and Exosomes for Tissue Regeneration. Scientific Reports, 2015. 5: p. 16662.

62. Mu, W., S. Rana, and M. Zöller, Host Matrix Modulation by Tumor Exosomes Promotes Motility and Invasiveness. Neoplasia, 2013. 15(8): p. 875-887.

63. Taylor, D.D. and C. Gerçel-Taylor, Tumour-derived exosomes and their role in cancer-associated T-cell signalling defects. British Journal of Cancer, 2005. 92: p. 305-311.

64. Peinado, H., et al., Melanoma exosomes educate bone marrow progenitor cells toward a prometastatic phenotype through MET. Nature Medicine, 2012. 18: p. 883-891.

65. Antonyak, M.A., et al., Cancer cell-derived microvesicles induce transformation by transferring tissue transglutaminase and fibronectin to recipient cells. Proc Natl Acad Sci U S A, 2011. 108(12): p. 4852-7.

66. Garnier, D., et al., Qualitative changes in the proteome of extracellular vesicles accompanying cancer cell transition to mesenchymal state. Experimental Cell Research, 2013. 319(17): p. 2747-2757.

67. Luga, V., et al., Exosomes Mediate Stromal Mobilization of Autocrine Wnt-PCP Signaling in Breast Cancer Cell Migration. Cell, 2012. 151(7): p. 1542-1556.

68. Costa-Silva, B., et al., Pancreatic cancer exosomes initiate pre-metastatic niche formation in the liver. Nat Cell Biol, 2015. 17(6): p. 816-26. 
69. Jung, T., et al., CD44v6 Dependence of Premetastatic Niche Preparation by Exosomes. Neoplasia, 2009. 11(10): p. 1093-1105.

70. Liu, Y., et al., Tumor Exosomal RNAs Promote Lung Pre-metastatic Niche Formation by Activating Alveolar Epithelial TLR3 to Recruit Neutrophils. Cancer Cell, 2016. 30(2): p. 243-256.

71. Kosaka, N., et al., Neutral sphingomyelinase 2 (nSMase2)-dependent exosomal transfer of angiogenic microRNAs regulate cancer cell metastasis. J Biol Chem, 2013. 288(15): p. 10849-59.

72. Hood, J.L., R.S. San, and S.A. Wickline, Exosomes Released by Melanoma Cells Prepare

Sentinel Lymph Nodes for Tumor Metastasis. Cancer Research, 2011. 71(11): p. 3792-3801.

73. Plebanek, M.P., et al., Pre-metastatic cancer exosomes induce immune surveillance by patrolling monocytes at the metastatic niche. Nat Commun, 2017. 8(1): p. 1319.

74. Yamamoto, S., et al., Inflammation-induced endothelial cell-derived extracellular vesicles modulate the cellular status of pericytes. Scientific Reports, 2015. 5: p. 8505.

75. Njock, M.-S., et al., Endothelial cells suppress monocyte activation through secretion of extracellular vesicles containing anti-inflammatory microRNAs. Blood, 2015.

76. Rautou, P.-E., et al., Microparticles From Human Atherosclerotic Plaques Promote Endothelial ICAM-1-Dependent Monocyte Adhesion and Transendothelial Migration. Circulation Research, 2011.

108(3): p. 335-343.

77. Mutschelknaus, L., et al., Radiation alters the cargo of exosomes released from squamous head and neck cancer cells to promote migration of recipient cells. Sci Rep, 2017. 7(1): p. 12423.

78. Arscott, W.T., et al., Ionizing Radiation and Glioblastoma Exosomes: Implications in Tumor Biology and Cell Migration. Translational Oncology, 2013. 6(6): p. 638-648.

79. Le, M., et al., Exosomes are released by bystander cells exposed to radiation-induced biophoton signals: Reconciling the mechanisms mediating the bystander effect. PLOS ONE, 2017. 12(3): p. $\mathrm{e} 0173685$.

80. Barile, L., et al., Extracellular vesicles from human cardiac progenitor cells inhibit cardiomyocyte apoptosis and improve cardiac function after myocardial infarction. Cardiovascular Research, 2014. 103(4): p. 530-541.

81. Vicencio, J.M., et al., Plasma Exosomes Protect the Myocardium From Ischemia-Reperfusion Injury. Journal of the American College of Cardiology, 2015. 65(15): p. 1525-1536.

82. Cantaluppi, V., et al., Microvesicles derived from endothelial progenitor cells enhance neoangiogenesis of human pancreatic islets. Cell Transplant, 2012. 21(6): p. 1305-20.

83. Lopatina, T., et al., Platelet-derived growth factor regulates the secretion of extracellular vesicles by adipose mesenchymal stem cells and enhances their angiogenic potential. Cell Communication and Signaling, 2014. 12(1): p. 26.

84. Tang, K., et al., Delivery of chemotherapeutic drugs in tumour cell-derived microparticles. Nature Communications, 2012. 3: p. 1282.

85. Shtam, T.A., et al., Exosomes are natural carriers of exogenous siRNA to human cells in vitro. Cell Communication and Signaling, 2013. 11(1): p. 88.

86. Cooper, J.M., et al., Systemic exosomal siRNA delivery reduced alpha-synuclein aggregates in brains of transgenic mice. Movement Disorders, 2014. 29(12): p. 1476-1485.

87. Alvarez-Erviti, L., et al., Delivery of siRNA to the mouse brain by systemic injection of targeted exosomes. Nature Biotechnology, 2011. 29(4): p. 341-345.

88. Elliman, S.J., J. Kavanaugh, and L. Couture, Sdc-2 exosome compositions and methods of isolation and use. 2017, Google Patents.

89. Elliman, S.J., Medical use of syndecan-2. 2014, Google Patents.

90. Vicente, C.M., et al., Syndecan-2 is upregulated in colorectal cancer cells through interactions with extracellular matrix produced by stromal fibroblasts. BMC Cell Biology, 2013. 14(1): p. 25.

91. Makler, A. and R. Narayanan, Mining Exosomal Genes for Pancreatic Cancer Targets. Cancer Genomics \& Proteomics, 2017. 14(3): p. 161-172.

92. Jakobsen, K.R., et al., Exosomal proteins as potential diagnostic markers in advanced non-small cell lung carcinoma. Journal of Extracellular Vesicles, 2015. 4: p. 10.3402/jev.v4.26659. 
93. Keller, S., et al., Body fluid derived exosomes as a novel template for clinical diagnostics. Journal of Translational Medicine, 2011. 9(1): p. 86.

94. Nilsson, J., et al., Prostate cancer-derived urine exosomes: a novel approach to biomarkers for prostate cancer. Br J Cancer, 2009. 100(10): p. 1603-1607.

95. Yang, C., et al., Comprehensive proteomics analysis of exosomes derived from human seminal plasma. Andrology, 2017. 5(5): p. 1007-1015.

96. Xu, J.-F., et al., Altered MicroRNA Expression Profile in Exosomes during Osteogenic

Differentiation of Human Bone Marrow-Derived Mesenchymal Stem Cells. PLoS ONE, 2014. 9(12): p. e114627.

97. Ogata-Kawata, H., et al., Circulating Exosomal microRNAs as Biomarkers of Colon Cancer.

PLOS ONE, 2014. 9(4): p. e92921.

98. Lener, T., et al., Applying extracellular vesicles based therapeutics in clinical trials - an ISEV position paper. Journal of Extracellular Vesicles, 2015. 4(1): p. 30087.

99. TRAIN-EV, Training in Extracellular Vesicles: for benefit in Health and Disease. Project ID: 722148. 2017, European Union under Horizon 2020.

100. Andrews, D.W., Pilot Immunotherapy Trial for Recurrent Malignant Gliomas. Retrieved from https://clinicaltrials.gov (Identification No. NCT01550523). 2012.

101. West, A., LRRK2 and Other Novel Exosome Proteins in Parkinson's Disease. Retrieved from https://clinicaltrials.gov/(Identification No. NCT01860118). 2013.

102. Saadatnia, M., Allogenic Mesenchymal Stem Cell Derived Exosome in Patients With Acute Ischemic Stroke. Retrieved from https://clinicaltrials.gov/ (Identification No. NCT03384433). 2017.

103. Curry, J., Metformin Hydrochloride in Mitigating Side Effects of Radioactive Iodine Treatment in Patients With Differentiated Thyroid Cancer. Retrieved from https://clinicaltrials.gov/ (Identification No. NCT03109847). 2017. 